\title{
SÍNTESE E CARACTERIZAÇÃO DA ZEÓLITA OFRETITA UTILIZANDO COGEL COMO FONTE DE SILIÍCIO
}

\author{
F. L. dos SANTOS ${ }^{1}$, A.O.S. da $\operatorname{SILVA}^{1}$, S. L. ALENCAR ${ }^{1}$, J. R. SANTOS ${ }^{1}$ e B. J. B. da \\ SILVA $^{1}$ \\ ${ }^{1}$ Universidade Federal de Alagoas, Centro de Tecnologia \\ E-mail para contato: fernandalima120@hotmail.com
}

\begin{abstract}
A zeólita Ofretita é de simetria hexagonal e suas estruturas podem ser descritas a partir de empacotamento de anéis de seis tetraedros. No presente trabalho foi desenvolvida a síntese e caracterização da zeólita Ofretita utilizando cogel como fonte de silício. As amostras obtidas foram caracterizadas por difratometria de raios X (DRX) e análises térmicas (TGA/DTG). Os resultados demonstraram que a zeólita Ofretita pode ser sintetizada com agitação mecânica em um período de $15 \mathrm{~h}$.
\end{abstract}

\section{INTRODUÇÃO}

Zeólitas são aluminossilicatos hidratados cristalinos, cujo arranjo estrutural é composto por um esqueleto formado pela combinação tridimensional de tetraedros de $\mathrm{AlO}_{4} \mathrm{e}$ $\mathrm{SiO}_{4}$, unidos entre si através de átomos de oxigênio (BRECK, 1974). São formadas por grande número de minerais sintéticos e naturais que possuem as mesmas características, onde são compostas por espaços intracristalinos que permite a transferência de matéria sendo limitada pelo diâmetro dos poros variando de uma zeólita para outra (LUZ, 1995).

Nestes espaços, estão localizadas as moléculas de água presentes na estrutura, assim como os cátions que neutralizam a carga negativa criada na estrutura pela presença do $\mathrm{AlO}_{2}{ }^{-}$. São colocados dentro dos canais microporosos alguns cátions como: os alcalinos $\left(\mathrm{Li}^{+}, \mathrm{Na}^{+}, \mathrm{K}^{+}, \mathrm{Rb}^{+}, \mathrm{Cs}^{+}\right)$, alcalinos-terrosos $\left(\mathrm{Mg}^{2+}, \mathrm{Ca}^{2+}, \mathrm{Ba}^{2+}\right), \mathrm{NH}^{4+}, \mathrm{H}_{3} \mathrm{O}^{+}, \mathrm{H}^{+}$e alguns cátions orgânicos contendo nitrogênio, íons de terras raras e metais nobres (SZOSTAK, 1998). Estes cátions conseguem se mover nos canais da rede cristalina da zeólita, podendo ser facilmente trocados por outros cátions de compensação.

A eficiência das zeólitas se deve a algumas características particulares desses materiais, entre elas: altas capacidades de adsorção, que variam desde altamente hidrofóbicas a altamente hidrofílicas; uma estrutura que permite a criação de sítios ativos, ácidos ou básicos, cuja força e concentração podem ser controladas de acordo com a aplicação desejada; tamanho de canais e cavidades compatíveis com a maioria das moléculas usadas na indústria; além de uma rede complexa de canais que lhes confere diferentes tipos de seletividade de forma (PAYRA e DUTTA, 2003).

Neste trabalho, a zeólita Ofretita foi sintetizada pelo método hidrotérmico com a utilização de cogel como fonte de sílica e em seguida foi caracterizada por difração de raios X e análise termogravimétrica para avaliar a obtenção da zeólita desejada. 


\subsection{Zeólita Ofretita}

A zeólita Ofretita possui fórmula química $\left|(\mathrm{Ca}, \mathrm{Mg})_{1,5} \mathrm{~K}\left(\mathrm{H}_{2} \mathrm{O}\right)_{14}\right|\left[\mathrm{Al}_{4} \mathrm{Si}_{14} \mathrm{O}_{36}\right]$-OFF (International Zeolite Association), é de simetria hexagonal com a sua estrutura descrita a partir do empacotamento de anéis de seis tetraedros e pela associação alternada de cavidades Cancrinitas e anéis duplos de seis membros. Essas associações geram colunas, as quais se unem entre si através de anéis duplos de seis simples tetraedros dando lugar às formações de cavidades, às quais se ingressa através de aberturas constituídas por anéis de oito átomos de oxigênio como mostra na Figura 1 (GIANNETTO, 2000).

Figura 1 - Estrutura da Zeólita Ofretita.
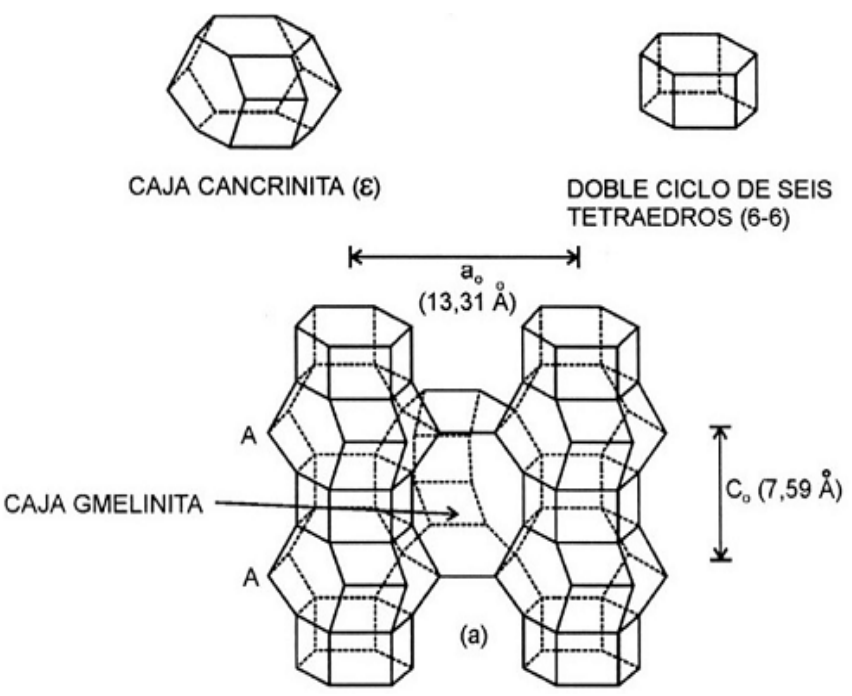

O empacotamento das cavidades Cancrinitas da zeólita Ofretita é do tipo AA, isso quer dizer que todas apresentam a mesma orientação, gerando um sistema de canais grandes, cilindros e paralelos, aos quais se penetra através de aberturas de doze átomos de oxigênio de diâmetro igual a $6,3 \AA$ que se comunicam entre si através de cavidades chamadas de Gmelinita, com aberturas formadas por anéis de oito átomos de oxigênio com diâmetro igual a 3,4 x 4,5 $\AA$ (GIANNETTO, 2000).

\section{EXPERIMENTAL}

Para a síntese da zeólita Ofretita, foram utilizados os reagentes apresentados na Tabela 1. 
Tabela 1 - Reagentes utilizados nas sínteses das amostras da zeólita Ofretita

\begin{tabular}{ll}
\hline \multicolumn{1}{c}{ Reagentes } & \multicolumn{1}{c}{ Fabricante/Características } \\
\hline Hidróxido de sódio & Merck, com teor de pureza de $75 \%$ de $\mathrm{Na}_{2} \mathrm{O}$ \\
\hline Hidróxido de potássio & Vetec, com teor de pureza de $71 \%$ de $\mathrm{K}_{2} \mathrm{O}$ \\
\hline Sulfato de alumínio hidratado & Merck, com teor de pureza de $51-59 \%$ \\
\hline Ácido sulfúrico & J.T. Baker, com teor de pureza de $98 \%$ \\
\hline Silicato de sódio neutro & $\begin{array}{l}\text { Sucroquímica Ind. e Com. Ltda, com teor de } \\
\text { pureza de } 11,2 \% \text { de } \mathrm{Na}_{2} \mathrm{O}\end{array}$ \\
\hline Água destilada & - \\
\hline
\end{tabular}

Primeiramente foi preparado o cogel da seguinte forma:

1) Dissolveu-se a fonte de alumínio em $70 \%$ de água requerido;

2) Adicionou-se o ácido sulfúrico (Solução A);

3) Diluiu-se o silicato de sódio nos restantes 30\% de água (Solução B);

4) Adicionou-se a solução A na solução B agitando por 60 minutos, até homogeneizar;

5) Em seguida, lavou-se por uma semana com água quente até a total retirada do sódio;

6) Centrifugou-se obtendo o cogel.

Posteriormente preparou-se o gel de síntese através do método hidrotérmico com a razão $\mathrm{SiO}_{2} / \mathrm{Al}_{2} \mathrm{O}_{3}$ do sistema $21, \mathrm{OH} / \mathrm{SiO}_{2}$ de $0,66, \mathrm{H}_{2} \mathrm{O} / \mathrm{SiO}_{2}$ de 21 e a de $\mathrm{K} / \mathrm{K}+\mathrm{Na}$ de 0,32 . $\mathrm{O}$ procedimento utilizado consiste das seguintes etapas:

1) Dissolução do hidróxido de potássio em metade de água para a síntese;

2) Em seguida, dissolução do hidróxido de sódio no restante de água;

3) Misturar as soluções dos itens anteriores;

4) Adição do cogel até a homogeneização.

Após a preparação do gel, uma parte deste foi transferido para um recipiente inoxidável de $1000 \mathrm{~mL}$ num reator Parr modelo 4520, sendo a mistura de reação agitada a $120 \mathrm{rpm}$ com um impulsor de hélice dupla, com seis lâminas cada rotor, enquanto o sistema era aquecido desde a temperatura ambiente até $150^{\circ} \mathrm{C}$. Quando cristalizado, o material sólido obtido foi separado do licor-mãe através de filtração a vácuo, foi lavado diversas vezes com água destilada quente até pH básico e seco, e colocado na estufa por 24 horas para secagem.

\section{RESULTADOS}

A difração de raios $\mathrm{X}$ foi a principal análise utilizada para a caracterização das amostras sintetizadas. Foi possível obter informações sobre o grau de cristalinidade das amostras e a presença de fases contaminantes.

$\mathrm{Na}$ Figura 2 são apresentados os difratogramas para as sínteses em condições dinâmicas. As amostras foram retiradas em diferentes tempos de cristalização (15h, 18h e 20h). As análises de difração de raios X apresentaram um material cristalino e de acordo com a estrutura padrão presente na literatura (ARIKA et al.,1987). 


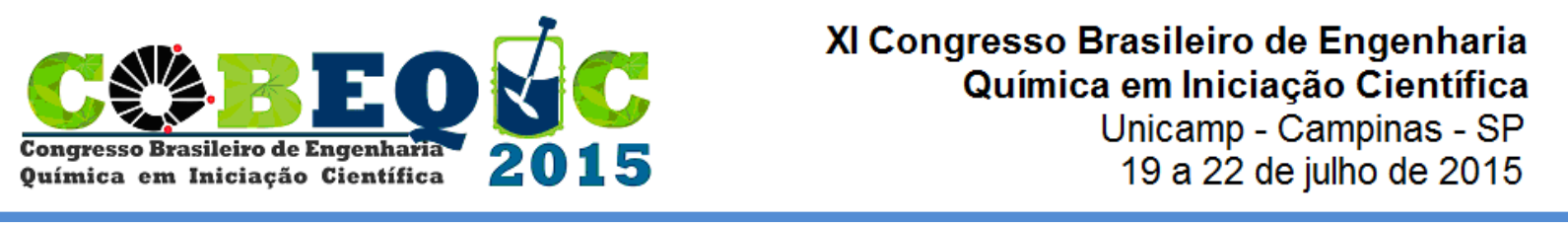

Figura 2 - Difratograma comparativo dos três tempos de cristalização em agitação mecânica.

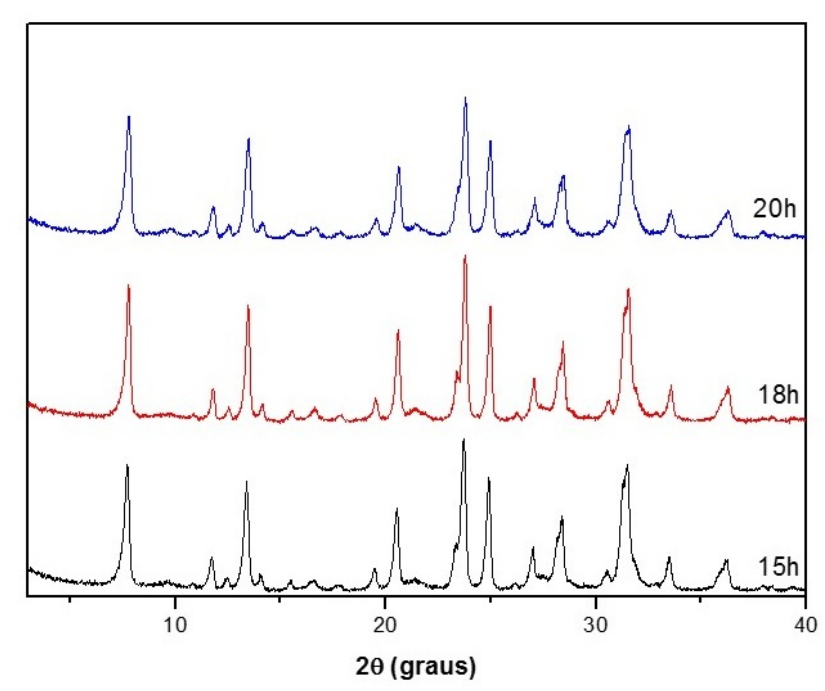

Estes dados indicaram que os sólidos obtidos nesta condição apresentam os picos de difração característicos da zeólita Ofretita com alta cristalinidade em apenas 15h. Esta cristalinidade foi calculada e mostrada na Tabela 2.

Tabela 2 - Cristalinidade das amostras sintetizadas

\begin{tabular}{cc}
\hline Amostra & $\begin{array}{c}\text { Cristalinidade } \\
(\mathbf{\%})\end{array}$ \\
\hline $15 \mathrm{~h}$ & 98 \\
\hline $18 \mathrm{~h}$ & 99 \\
\hline $20 \mathrm{~h}$ & 100 \\
\hline
\end{tabular}

A amostra de 20 horas apresentou o maior valor correspondente ao somatório das áreas sob os picos selecionados $\left(2 \theta=22,68^{\circ}\right.$ e $\left.25,64^{\circ}\right)$ e por este motivo foi considerada $100 \%$ cristalina.

A análise termogravimétrica da zeólita Ofretita sintetizada em condições dinâmicas e com tempo de cristalização de 20 horas, encontra-se apresentada na Figura 3, onde tem como principal característica dois eventos distintos de perda de massa. 
Figura 3 - TGA e DTG da amostra da zeólita Ofretita mais cristalina.

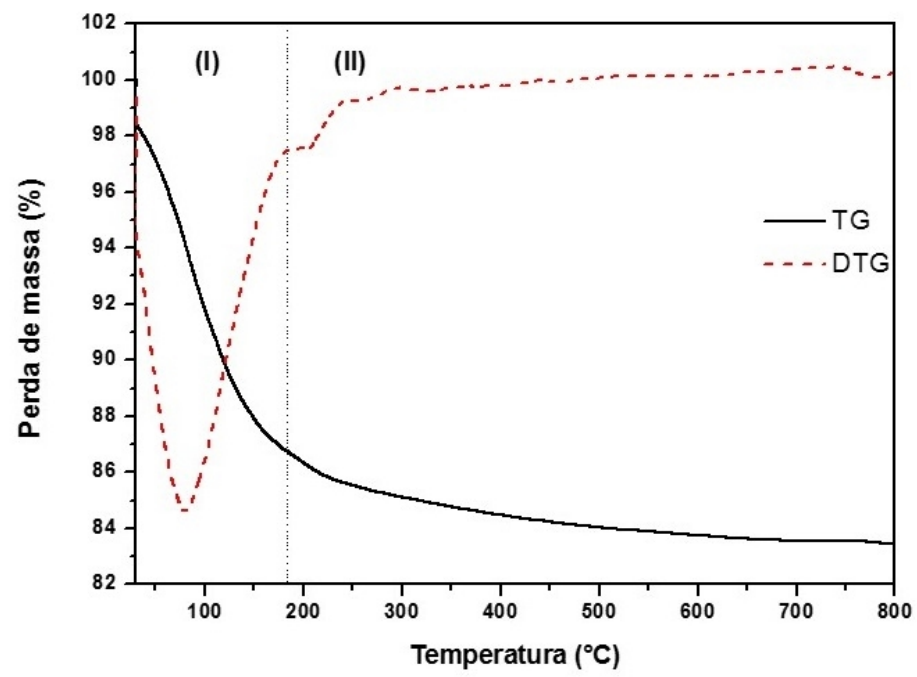

De acordo com a literatura (ARAÚJO, 2006; SILVA, 2004), estes eventos foram atribuídos aos seguintes processos: 1) a desidratação da zeólita sintetizada, com perda de massa de $11,65 \%$ e 2) a perda de água estrutural devido a condensação do grupo silanol (composto em que uma hidroxila está ligada a um átomo de silício), com perda de 3,28\%. Confirmando que seus picos estão essencialmente relacionados com o tipo de variação de massa.

\section{CONCLUSÃO}

A zeólita Ofretita foi realizada através do método hidrotérmico utilizando cogel como fonte de sílica. Com base nos resultados obtidos pode-se concluir que:

- Os difratogramas de raios $\mathrm{X}$ da zeólita Ofretita apresentaram os picos característicos da estrutura com agitação mecânica no tempo de cristalização, indicando um elevado grau de cristalinidade;

- As curvas TGA/DTG da zeólita Ofretita obtidas após a síntese hidrotérmica, indicam que há a desidratação da zeólita sintetizada e a perda de água estrutural em dois eventos.

- A curva DTG da zeólita Ofretita apresenta um pico endotérmico na região entre 30 e $170{ }^{\circ} \mathrm{C}$, característico de um processo de desidratação.

- A formação da zeólita Ofretita foi apenas possível com agitação no processo de cristalização. 


\section{REFERÊNCIAS}

ARAÚJO, A.S., Desenvolvimento de catalisadores zeolíticos destinados à reforma do metano. 2006. 153 f. Tese (Doutorado em Engenharia de Processos) - Programa de PósGraduação em Engenharia de Processos, Universidade Federal de Campina Grande, Campina Grande, 2006.

ARIKA, J., IGAWA, K., ITABASHI, K., Process for Preparation of Zeolite OE having an Offretite type Structure, US Patent 4.687.653, 1987.

BRECK, D.W., Zeolite Molecular Sieves, Structure, Chemistry and Use, Wiley, New York, 1974.

GIANNETTO, G. P. Zeolitas - Características, propiedades y aplicaciones industriales. Editorial Innovación Tecnológica, Caracas, 2000. 170.

GONZAGA, A. C. Desenvolvimento de nanocatalisadores (Fe/ZSM-5) destinados a Reação de Fisher-tropsch. Monografia (Programa de Recursos Humanos da Agência Nacional de Petróleo e Gás PRH-25), Centro de Ciências e Tecnologia, Universidade Federal de Campina Grande, PB, 2007.

LUZ, A. B., "Zeólitas: Propriedades e usos industriais", 1ª edição, CETEM/ CNPq, (Série Estudos e Documentos; 68), Rio de Janeiro, 1995.

PAYRA, P.; DUTTA, P. K. in: Auerbach, S. M. (Ed); Carrado, K. A. (Ed); Dutta, P. K. Handbook of Zeolite Science and Technology. New York: Marcel Dekker, Capítulo 1, Zeolites: A Primer, 1184f., 2003.

SILVA, A.O.S. Síntese e Caracterização de Catalisadores de Ferro e Cobalto Suportados nas Zeólitas HZSM-12 e HZSM-5 para a Conversão de Gás de Síntese em Hidrocarbonetos. 2004. 229 f. Tese (Doutorado em Engenharia Química) - Programa de Pós-Graduação em Engenharia Química, Universidade Federal do Rio Grande do Norte, Natal/RN, 2004.

SZOSTAK, R. Molecular Sieves: Principles of Synthesis and Identification. Blackie Academic \& Professional, 2a Ed. New York: Van Nostrand Reinhold, 60, 1998. 